\title{
Freedom in computing : Libreboot, Panfrost report.
}

\author{
Camille Akmut
}

\begin{abstract}
A report on a free boot firmware, Libreboot, and a free GPU driver for ARM computers, Panfrost; with additional information on coreboot and Tamil/Lima. Companion publication to: "When we liberate ourselves we start from the bottom. Introduction to an upcoming report..."
\end{abstract}




\section{Foreword : Freedom in computing}

In the present report, the term "free" is found in abundance : "a free boot firmware" says the official documentation for Libreboot, while Panfrost is similarly described "a free driver".

Both of these designations go back to Richard Stallman, the concept of "free software" :

Free software is software that gives (...) the user the freedom to share, study and modify it. (...)

To use free software is to make a political and ethical choice asserting the right to learn, and share what we learn ${ }^{1}$

"I strongly believe in Free Software. I have greatly admired Richard Stallman and his ilk since I was very young (basically as soon as I started using computers regularly, when I first heard about GNU+Linux)." wrote Leah Rowe, creator of Libreboot ${ }^{2}$.

"At home, I discovered writings by Richard Stallman, the father of the free software movement. I was mesmerised." wrote Alyssa Rosenzweig similarly, creator of Panfrost ${ }^{3}$.

"Please use your programming capability only for good, not for evil. Don't develop nonfree software, or service as a software substitute. Design systems not to collect personal information, and to allow anonymous use." wrote Richard Stallman himself, in his seminal "How I do my computing" $\operatorname{text}^{45}$.

Both projects, and creators, not only follow their guide, but are at the forefront of what we call here "Freedom in computing" generally, and Rosenzweig calls more precisely "low-level freedom" : the deepest parts of our computers, that are those that remain least known to us - in this case various pieces of software, called "firmware" here and "driver" there, used variously for booting, to graphics ${ }^{6}$.

"Tools unknown live lives of their own", we wrote elsewhere; Bachelard not far away, nested somewhere in the back of our mind. This makes such projects all the more important : vital even - if not quite now, sooner or later.

But, better sooner than later, and "better premature than belated" as we wrote still at the very beginning of our inquiries into the history of computer science and technology : our computers, for now, still stand on our desks, rest on our laps, some of them have gotten as far as our pockets in this early century, and a minority for now under our skin (microchip).

One does not need a sound understanding of history, to predict in what direction computers are going. The answer is : us.

\footnotetext{
${ }^{1}$ Free Software Foundation 2019.

${ }^{2}$ Rowe 2019.

${ }^{3}$ Rosenzweig 2017.

${ }^{4}$ Stallman 2019.

${ }^{5}$ A tradition furthered in, amongst others, the previously quoted Rowe 2019, and (arguably) Rosenzweig 2018b.

${ }^{6}$ So much work remains to be done despite all that has already been accomplished. This includes for instance the need for free wifi drivers; a field whose state appears catastrophic...
} 


\section{Libreboot : a free boot firmware, BIOS/UEFI replacement}

The official description for Libreboot reads :

Libreboot is a free boot fi[r]mware implementation, to replace the non-free BIOS/UEFI firmware found on most systems. It initializes the hardware and starts a bootloader for your operating system. ${ }^{7}$

To better understand this, we can (and should) make use of the existing literature on operating systems :

A simplified view of the boot process looks like this:

1. The machine's BIOS or boot firmware loads and runs a boot loader.

2. The boot loader finds the kernel image on disk, loads it into memory, and starts it.

3. The kernel initializes the devices and its drivers.

4. The kernel mounts the root filesystem.

5. The kernel starts a program called init with a process ID of

1. This point is the user space start.

6. init sets the rest of the system processes in motion.

7. At some point, init starts a process allowing you to log in, usually at the end or near the end of the boot. ${ }^{8}$

A more precise definition for the boot loader is given so : "It loads the kernel into memory, and then starts the kernel with a set of kernel parameters." "On PCs, boot loaders use the Basic Input/Output System (BIOS) or Unified Extensible Firmware Interface (UEFI) to access disks." 9

Thus, following this schema, Libreboot fullfills 1., boot firmware, and 2., boot loader (and possibly some of 3., device drivers). Crucially, it does so as a program whose nature permits "to share, study and modify it", based on the already quoted definition of free software, and contrary to existing, dominant, closed BIOS/UEFI.

The project was started in December 2013 and initially only supported the ThinkPad X60 computer $^{10}$, it has now grown to support the X200 and T400 model of the same - among others ${ }^{11}$.

Going into more detail, the documentation further notes :

Libreboot's main upstream providers are coreboot (which we deblob, for hardware initialization), depthcharge (bootloader, and default libreboot payload on ARM), and GRUB (bootloader, and default libreboot payload on x86). We also integrate flashrom (for installing libreboot), and several of our own

\footnotetext{
${ }^{7}$ https://notabug.org/libreboot/libreboot

${ }^{8}$ Ward 2015 : 93-94. The more academically-minded textbooks do not provide more insight, but alternatively Tanenbaum or Silberschatz can be consulted.

${ }^{9}$ Ibid : 97.

${ }^{10}$ Rowe 2017.

${ }^{11} \mathrm{~A}$ full list can be found here : https://libreboot.org/docs/hardware/
} 
utilities, scripts and configuration files. All of this is integrated into a single, coherent package that is easy to use. We add our own patches to the various upstreams used, and where feasible try to merge upstream as much as possible.

Together, Libreboot's build system and documentation is provided with the aim of making free boot firmware accessible to all. $^{12}$

\section{Panfrost : a free GPU driver for ARM computers}

Panfrost takes a different approach, and makes a different bet : rejecting the $\mathrm{x} 86$ architecture, prefering to concentrate on ARM, RISC instead.

In her own words, Alyssa Rosenzweig describes the project and her way toward it so :

I have developed a focus on low-level freedom. I joined Libreboot, a free boot firmware, and through that immersion in boot freedom, I learned of two grave new threats: the Intel Management Engine and the AMD Platform Security Processor. It became clear that Intel and AMD's x86, the dominant architecture among free and proprietary software users alike, no longer belonged in our movement. I switched to ARM machines.

Unfortunately, free software support for ARM is lacking. On popular almost-free chipsets like the RK3288, the graphics processor requires proprietary blobs. Thus, we hackers are creating Panfrost, a free driver for modern ARM Mali chips. Today, on an RK3288 laptop, Panfrost is mature enough to run the famous benchmark, es2gears, with zero lines of proprietary code. But even with projects like Panfrost, intense ARM fragmentation has made the architectural jump a RISCy proposition for free software supporters. Indeed, there is not yet a userfriendly, fully free GNU/Linux distribution available for ARM. (...) Longer-term, I seek to improve distribution support to enable x86-bound users to make the switch. No one - and no zero - has claimed the road ahead is easy. But little by little, together we can chip away at the proprietary monopoly, in the name of freer chips. ${ }^{13}$

In an earlier post, she gave more details :

Over the summer I founded the chai project. My goal was to write a free software driver for Midgard GPUs, better known as the Mali T series produced by ARM. Why? Libreboot supports the Rockchip RK3288 chipset, containing a Mali T760 GPU

\footnotetext{
12 https://notabug.org/libreboot/libreboot

${ }^{13}$ Rosenzweig $2018 \mathrm{c}$.
} 
that is unusable without proprietary software. I purchased a laptop [An Asus C201 Chromebook made in 2015] with this chipset, and thus my work began. ${ }^{14}$

In searches for definitions of "proprietary blobs", the following Free Software Foundation GNU documentation can (and should) be considered a relevant, pertinent source :

"The kernel that they distribute (in most cases, Linux) includes "blobs": pieces of object code distributed without source, usually firmware to run some device." ${ }^{15}$

The "famous benchmark, es2gears" mentioned has the following man (manual) entry in GNU/Linux systems :

$\$$ man es2gears

es2gears - "'gears') demo for GLES2

DESCRIPTION

The es2gears program is a port of the "gears') demo to GLES2. It displays a set of rotating gears and prints out the frame rate at regular intervals. It has become quite popular as basic benchmarking tool.

Where GLES2 stands for perhaps better known OpenGL ES 2, a subset of the OpenGL graphics API ${ }^{16}$ for embedded systems (hence its acronym of "ES", and hence also the name of this demo/benchmarking tool).

It can be installed like any other program :

\$ es2gears

Command 'es2gears' not found, but can be installed with:

sudo apt install mesa-utils-extra

In figure 1, Lyude Paul, the other main developer of the project, together with Rosenzweig execute the program ./panfrost es2gears successfully on an ARM computer with a modern GPU, what appears to be the ROCKPro64 (Rockchip RK3399 with Mali T860 MP4 GPU ${ }^{17}$ ).

Panfrost had precursors in Tamil/Lima, just as Libreboot had coreboot, started 1999, for antecedent :

I was not the first to free the GPU. Luc Verhaegan, better known by his handle libv, precedes me. His project, Tamil, reverse engineered enough of the command stream2 to display a textured cube at FOSDEM.

But the project ceased there due to the pushback; he never released his work. ${ }^{18}$

\footnotetext{
${ }^{14}$ Rosenzweig 2018.

${ }^{15}$ GNU 2019.

${ }^{16}$ Successor : Vulkan.

${ }^{17}$ Based on the manufacturer's specifications : https://www.pine64.org/rockpro64/

${ }^{18}$ Rosenzweig 2018.
} 


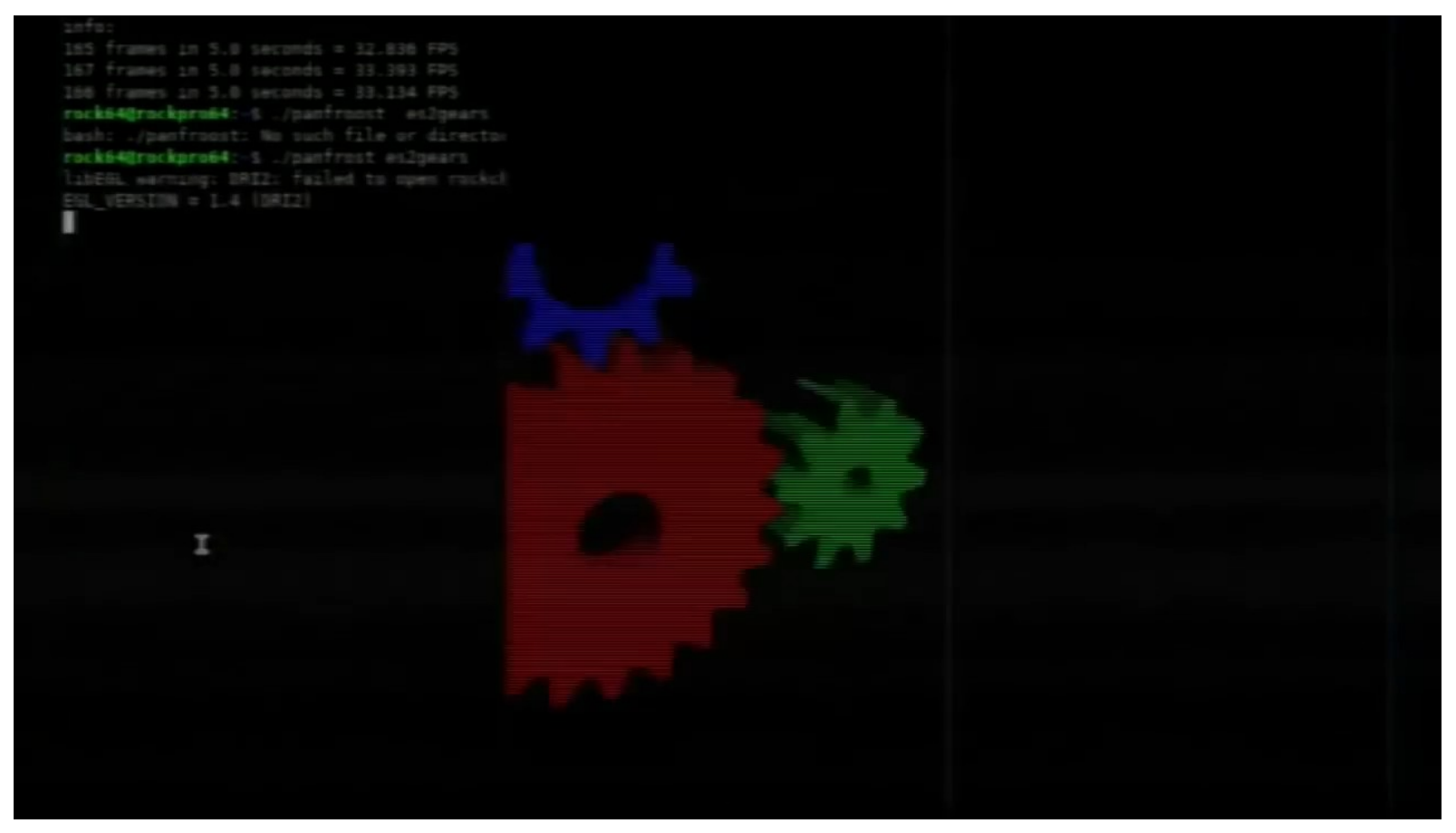

Figure 1: Paul and Rosenzweig 2018.

She may be referring to his appearance at FOSDEM 2013, demonstrating the Lima driver ${ }^{19}$, whose description reads :

When the Lima driver was presented at last years FOSDEM, it was a game changing moment. Before this reverse engineering project for the highly popular ARM Mali 200/400 GPUs was announced, the open source graphics driver world was fully focused on the three x86 market leaders only, and the huge ARM market was being completely ignored (from a graphics driver point of view). The Lima driver fundamentally changed this, and now open ARM graphics drivers are a hot topic, and several people have taken it upon themselves to follow the Lima example. ${ }^{20}$

Elsewhere, he writes :

Due to my usual combination of stubbornness and actually sitting down and doing the work, I have personally kickstarted the whole open ARM GPU movement. This is the third enormous paradigm shift in Linux graphics that i have caused these past almost 12 years (after modesetting, and pushing ATI to a

\footnotetext{
${ }^{19}$ Verhaegen 2013.

${ }^{20}$ Ibid.
} 
point of no return with an open source driver). All open ARM

GPU projects were at least inspired by this, some actually use some lima code, others would simply not have happened if I hadn't done Lima ${ }^{21}$

\section{References}

—. 2018 [2017]. "Social conditions of outstanding contributions to computer science : a prosopography of Turing Award laureates (1966-2016)."

—. 2019. "'Average intellectuals' and the Middle ages : an introduction with translation, and apparatus."

Free Software Foundation. 2019. "What is free software and why is it so important for society?". https://www.fsf .org/about/what-is-free-software

GNU. 2019. "Explaining Why We Don't Endorse Other Systems". https : //www.gnu.org/distros/common-distros.html

Libreboot. https://notabug.org/libreboot/libreboot

Paul, Lyude and Rosenzweig, Alyssa. 2018. "Lyude Paul \& Alyssa Rosenzweig - Introducing Panfrost". X.Org Developer's Conference. https: //www $\cdot$ youtube. com/watch?v=qtt2Y7XZS3k

Rosenzweig, Alyssa. 2017. "Growing Up to Free Software". 11/05. https://rosenzweig.io/blog/growing-up-to-free-software.html

-. 2018. "My Name is Cafe Beverage". 18/01. https://rosenzweig. io/blog/my-name-is-cafe-beverage.html

—. 2018b. "Living On A Rockchip". 05/04. https://rosenzweig.io/ blog/living-on-a-rockchip.html

-. 2018c. "Introducing Alyssa Rosenzweig, intern with the FSF tech team". 12/07. https://www.fsf.org/blogs/sysadmin/introducing-alyssa-rosenzweig-intern-with-the-fs

Rowe, Leah. 2019. "How I do my computing". 28/04. https://blog. vimuser.org/leah-computing.html

Rowe, Leah. 2017. "Libreboot Free your BIOS today!". FOSDEM. https://www . youtube.com/watch?v=W1ta--tHenE

Stallman, Richard. 2019. "How I do my computing". https://stallman. org/stallman-computing.html

Verhaegen, Luc. 2013. "[FOSDEM 2013] Open ARM GPU drivers". FOSDEM. https : //www . youtube . com/watch?v=7z6xjIRXcp4

-. 2015. "The value of writing code and instigating change." 23/04. https://libv.livejournal.com/27461.html

Ward, Brian. 2015. How Linux Works. No Starch Press. (In particular Chapter 5 : "How the Linux Kernel Boots".)

${ }^{21}$ Verhaegen 2015. 
Acknowledgement ("I'd like to send this one out for...")

Abel/Alexina/Camille/Herculine Barbin (1838-1868),

Lili Elbe (1882-1931),

Dora "Dörchen" Richter (1891-1933),

Lynn Conway (1938-),

Wendy Carlos (1939-),

Lou Reed (1942-2013) and Rachel (?-?),

Candy Darling (1944-1974),

Marsha P. Johnson (1945-1992),

Leslie Feinberg (1949-2014),

Sylvia Rivera (1951-2002),

Mary Ann Horton (1955-),

Lana (1965-) and Lilly Wachowski (1967-),

Aya Kamikawa (1968-),

Audrey Tang (1981-),

Cash Askew (?-2016), of Them Are Us Too, who lived to be 22,

Rachel Bryk (?-2015), Dolphin emulator developer, a year more, 23,

Chloe Sagal (?-2018), game developer, Homesick, just some more, 31,

Chelsea Elizabeth Manning (1987-),

Bailey Jay (1988-),

Andreja Pejic (1991-),

Sasha "Scarlett" Hostyn (1993-),

And, all the anonymous ones whose up to real names we don't even know, doubly erased and robbed, from us, and themselves, in life, and in death, once more, and again.

If we don't tell our stories, they never will - or, worse perhaps, they will, and they will do us wrong, like Foucault possibly did to Barbin (with good intentions too), along with the rest of her/their/his commentators, doctors, judges, police, psychiatrists, psychologists, journalists, "gender theorists", philosophers, (medical, or science) historians, or else, etc. etc.. 